\title{
Power Minimization Under Throughput Management Over Wireless Networks With Antenna Diversity
}

\author{
Zhu Han, Member, IEEE, and K. J. Ray Liu, Fellow, IEEE
}

\begin{abstract}
In multiaccess wireless communication systems, power control and adaptive modulation are two important means to increase spectral efficiencies, combat time-varying fading channels, and reduce cochannel interferences. The overall uplink transmitted power is minimized under the constraints that there is no reduction in overall network throughput and each user achieves the desired time-average throughput. Adaptive M-QAM modulations with two kinds of antenna diversity are considered. Each user can select a range of modulation rates, according to his channel condition and transmission history. Two subproblems are considered for the development of suboptimal low-complexity adaptive algorithms. First, at the user level, the following needs to be determined: the range of modulation rates that each user can accept at a specific time to ensure fairness. Then, each time at the system level, within the acceptable ranges, the system finds out what throughput allocation for different users requires the lowest overall transmitted power. The scheme can be interpreted as "water filling" each user's throughput in time domain and allocating network throughput to different users at each time. From the simulation results, the proposed scheme reduces the overall transmitted power up to $7 \mathrm{~dB}$ and increases average spectral efficiency up to $1.2 \mathrm{bit} / \mathrm{s} / \mathrm{Hz}$, compared with the previous known power control schemes.
\end{abstract}

Index Terms-Antenna arrays, power control, resource management.

\section{INTRODUCTION}

$\mathbf{I}$ $\mathrm{N}$ WIRELESS communication systems, two important detrimental effects to decrease the network performance are the channel's time-varying nature and cochannel interference (CCI). Because of the effects such as multipath fading, shadowing, path loss, propagation delay, and noise level, the signal-to-interference-noise-ratio (SINR) at a receiver output can fluctuate in order of tens of decibels. Since the available bandwidth is limited, the channels are reused for different transmissions, which causes CCI. A general strategy to combat these detrimental effects is the dynamic allocation of resources, such as transmitted powers and modulation rates, based on the channel conditions. In power control, transmitted powers are constantly adjusted. Such a process improves the qualities of weak links. At the same time, it increases CCI during deep fading. In adaptive modulation, the system assigns modulation rates with different constellation sizes and spectral efficiencies to different links, according to their channel conditions. There

Manuscript received October 18, 2002; accepted September 6, 2003. The editor coordinating the review of this paper and approving it for publication is A. Svensson.

The authors are with the Electrical and Computer Engineering Department, Institute for Systems Research, University of Maryland, College Park, MD 20742 USA (e-mail: hanzhu@glue.umd.edu; kjrliu@glue.umd.edu).

Digital Object Identifier 10.1109/TWC.2004.837431 are tradeoffs and practical constraints to allocate these resources. How to efficiently manage these resources has become an important research issue.

Much work has been done for resource allocation, such as power control and adaptive modulation in multiaccess wireless channels. In [1]-[4], resource allocation has been extensively studied. In [5]-[8], beamforming, multiuser detection, and power control have been combined for cellular wireless communication systems. In [9]-[15], adaptive modulation techniques have been proposed to enhance the spectrum efficiency for wireless channels. The performance approximation and robustness for estimation errors have been investigated. In [16] and [17], adaptive coding provides another way for transmission rate control. In [18]-[20], the authors have explored resource allocation problems from the channel capacity point of view. In [21]-[28], many adaptive algorithms are constructed to adaptively control the transmitted power and rate to optimize the system performance. In [29], the authors present an "opportunistic" transmission scheduling policy for a single cell time-division multiple access/frequency-division multiple access (TDMA/FDMA) system that exploits time-varying channels and maximizes the system performance stochastically. In [30], game theory is introduced in the power control problem. Each user competes with other users for limited resources, and the system is balanced in some equilibriums.

In traditional power control, each link's transmitted power is selected so that its received SINR is larger than or equal to a fixed and predefined targeted SINR threshold, required to maintain its link quality, while the system minimizes the overall transmitted power of all links. However, a link with a bad channel condition requires too much transmitted power and therefore causes unnecessary CCI to other links. This is a major issue that will be addressed in this paper. In adaptive modulation, each link can select a range of different modulation rates; consequently, a range of targeted SINR thresholds can be applied. A joint adaptive power and rate allocation scheme is developed by using M-QAM adaptive modulation with antenna diversity. The optimization goal is to minimize the overall transmitted power under some constraint. The overall network throughput is not reduced; the time-average throughput of each user is maintained as a constant that is determined by the service for which the user pays. In order to solve the problem, the problem is heuristically divided into two subproblems. First, users determine the ranges of throughput that they can accept at different times and report these ranges back to the system. An algorithm is developed to ensure fairness at the user level. Second, the system determines what is the optimal throughput allocation to different users at each time within 
the acceptable throughput ranges provided by the users. Three adaptive algorithms are developed to solve this subproblem at the system level. The whole scheme can be interpreted as "water filling" each user's throughput in time domain and allocating network throughput to different users each time, according to their channel conditions. From the simulation results, the proposed scheme reduces the overall transmitted power up to $7 \mathrm{~dB}$ and increases the average spectral efficiency up to $1.2 \mathrm{bit} / \mathrm{s} / \mathrm{Hz}$, compared with the previous scheme in [5].

The organization of this paper is as follows: In Section II, a network system model is presented with antenna diversities. Approximations of MQAM are presented. The optimization problem is formulated. In Section III, the problem is heuristically divided into two subproblems. Several adaptive algorithms are developed. A power and throughput management system is constructed. In Section IV, the proposed algorithms are evaluated by simulation study. In Section V, the conclusion is presented.

\section{System Model AND PROBLEM Formulation}

\section{A. System Model}

$K$ cochannel links exist in distinct cells, such as in TDMA or FDMA networks. Each link consists of a mobile unit and its assigned base station. Coherent detection is assumed to be possible so that it is sufficient to model this multiuser system by an equivalent baseband model. Antenna arrays with $P$ elements are used only at the base stations. Each link is affected by the multipath fading, with the propagation delay far less than one symbol duration. The maximum number of paths is $L$. For the uplink case, the signal at the $p$ th antenna array element of the $i$ th base station can be expressed as

$x_{i}^{p}(t)=\sum_{k=1}^{K} \sum_{l=1}^{L} \sqrt{\rho_{k i} G_{k i} P_{k}} \alpha_{k i}^{p l} g_{k}\left(t-\tau_{k i}\right) s_{k}\left(t-\tau_{k i}\right)+n_{i}^{p}(t)$

where $\rho_{k i}$ and $G_{k i}$ are the log-normal shadow fading and the path loss from the $k$ th user to the $i$ th base station, respectively, $\alpha_{k i}^{p l}$ is the $l$ th path fading loss from the $k$ th user to the $i$ th base station's $p$ th antenna, $P_{k}$ is the transmitted power, $g_{k}(t)$ is the shaping function, $s_{k}(t)$ is the message symbol, $n_{i}^{p}(t)$ is the $i$ th base station's thermal noise at the $p$ th antenna, and $\tau_{k i}$ is the channel propagation delay. Here, $\tau_{i i}=0, \forall i$ (the delay from the mobile to its assigned BS), and $\tau_{k i}, k \neq i$ (the delay from the mobile to other cell's BS) is uniformly distributed within one symbol duration. The channels change slowly and are stable over a frame with hundreds of symbols. The impulse response from the $k$ th mobile to the $p$ th element of the $i$ th base station is defined as: $h_{k i}^{p}=\sum_{l=1}^{L} \alpha_{k i}^{p l} r_{k i}^{p}$, where $r_{k i}^{p}$ includes the effects of the transmitter, receiver filter, and shaping function $g_{k}(t-$ $\left.\tau_{k i}\right)$. We define $n_{i}^{p}(n)$ as the sampled noise.

Because of the channel distortions, CCI, and thermal noises, the average receivers' SINR can be very low most of the times. Under this condition, in order to satisfy the desired bit-error rate (BER), only low modulation rate or even no transmission can be selected. Antenna diversity is an important means to increase the average receiver's SINR. Consequently, MQAM can be applied with different modulation rates for the desired BER. The antenna outputs can be combined by maximal ratio combining (MRC) or selective combining (SC) [31], as shown in Fig. 1. MRC diversity requires that the individual signals from each branch be compensated in phase and weighted by the square roots of their SINRs and then be summed coherently. If perfect knowledge of the branch amplitudes and phases is assumed, when the noise is spatially white, MRC is the optimal diversity-combining scheme and provides the maximum capacity improvement. The disadvantage of MRC is that it requires all knowledge of the branch parameters. The SC combiner only chooses the branch with the highest SINR. SC is simpler than MRC but yields suboptimal performance. By using the antenna diversity, the $i$ th base station's combiner output can be written as $\mathbf{w}_{i}^{H} \mathbf{x}_{i}$, where $\mathbf{x}_{i}=\left[x_{i}^{1} \ldots x_{i}^{P}\right]^{T}$, and $\mathbf{w}_{i}$ is a $P \times 1$ combiner weight vector given by

$$
\left\{\begin{array}{l}
\text { For MRC: } \quad\left|\left[\mathbf{w}_{i}\right]_{j}\right|=\sqrt{\Gamma_{i}^{j}} \\
\text { For SC: }\left[\mathbf{w}_{i}\right]_{j}= \begin{cases}1, & j \text { antenna has the largest SINR } \\
0, & \text { otherwise }\end{cases}
\end{array}\right.
$$

where $\Gamma_{i}^{p}$ is the received SINR at the $p$ th antenna element that can be calculated from (1). The $i$ th base station's combiner output SINR is given by [26]

$$
\Gamma_{i}=\frac{P_{i} \rho_{i i} G_{i i}\left\|\mathbf{w}_{i}^{H} \mathbf{h}_{i i}\right\|^{2}}{\sum_{k \neq i} P_{k} \rho_{k i} G_{k i}\left\|\mathbf{w}_{i}^{H} \mathbf{h}_{k i}\right\|^{2}+\mathbf{w}_{i}^{H} \mathbf{N}_{i} \mathbf{w}_{i}}
$$

where $\mathbf{h}_{k i}=\left[h_{k i}^{1}, \ldots, h_{k i}^{P}\right]^{T}, \mathbf{N}_{i}=E\left\{\mathbf{n}_{i} \mathbf{n}_{i}^{H}\right\}$, and $\mathbf{n}_{i}=$ $\left[n_{i}^{1} \ldots n_{i}^{P}\right]^{T}$.

In adaptive modulation, the transmitters and receivers can adaptively select the modulation rates, i.e., throughput, according to the channel conditions. It has been shown that adaptive modulation can greatly increase the spectral efficiency of wireless communications [10], [22]. In this paper, adaptive MQAM modulation is applied. It has been shown that BER of square MQAM with gray bit mapping as a function of received SINR $\Gamma$ and constellation size $M$ is approximately given by [31]

$$
\operatorname{BER}(\Gamma, M) \approx \frac{2}{\log _{2} M}\left(1-\frac{1}{\sqrt{M}}\right) \operatorname{erfc}\left(\sqrt{1.5 \frac{\Gamma}{M-1}}\right)
$$

where erfc is the complementary error function. This approximation is tight when the SINR $\Gamma$ is high.

Now the relation between SINR and throughput will be shown. In the $i$ th cell, the $i$ th link between the mobile and its assigned base station uses the modulation with constellation size $M_{i}$. Without loss of generality, each user is assumed to have the unit bandwidth. The $i$ th link has throughput $T_{i}=\log _{2}\left(M_{i}\right)$. For BER $=10^{-2}$ and BER $=10^{-5}$, the required SINRs of different constellation sizes are shown in Fig. 2. One can see that for the traditional power control with fixed modulation (8-QAM), the receiver must have SINR greater than a specific threshold to have any throughput that satisfies BER $=10^{-5}$. In this paper, each user can select a range of different modulation 

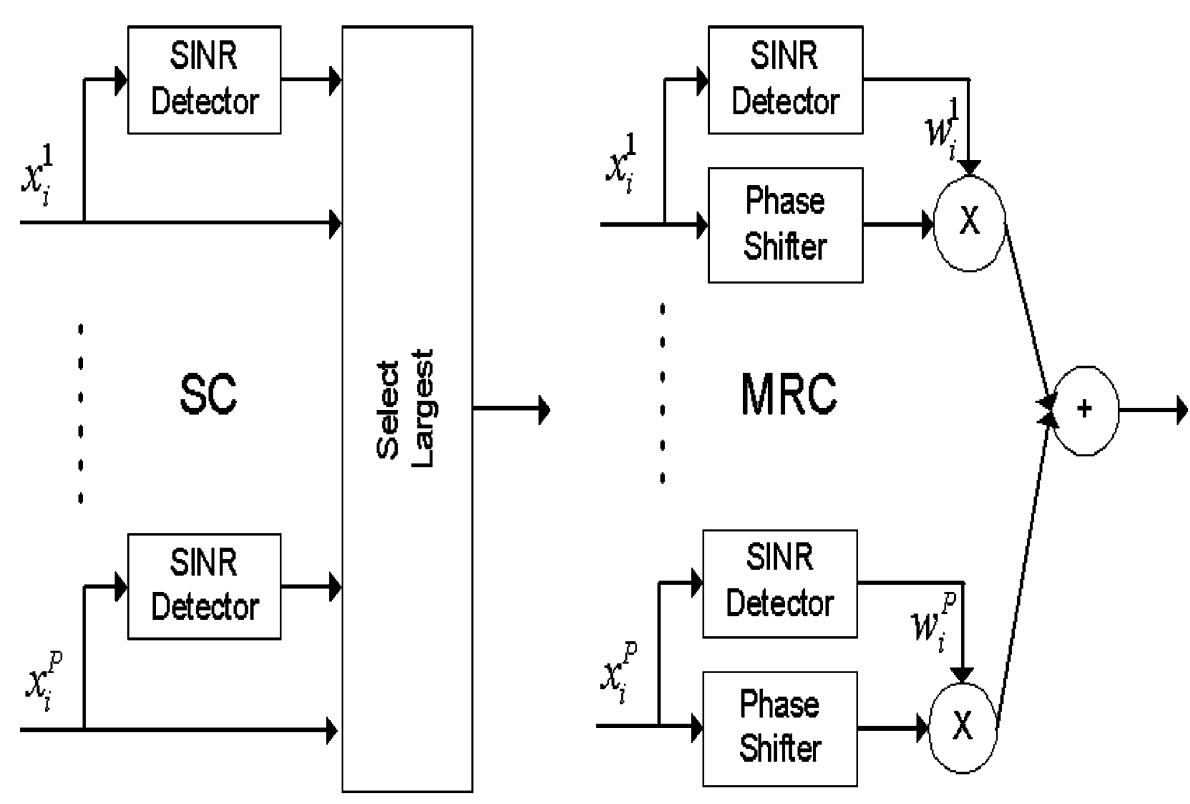

Fig. 1. SC and MRC.

rates. Consequently, the targeted receiver's SINR can be chosen within a range.

It is hard for (3) to be inverted and differentiated. In [10] and [22], the authors have introduced BER approximations for different modulation rates as

$$
\mathrm{BER}_{i} \approx c_{1} e^{-c_{2} \frac{\Gamma_{i}}{{ }^{c_{3} T_{i}}-1}}
$$

where $c_{1} \approx 0.2, c_{2} \approx 1.5$, and $c_{3} \approx 1$. This approximation is tight when the SINR is high. Rearranging (4) for a specific $\mathrm{BER}$, the $i$ th link's throughput is given by

$$
T_{i}=c_{4}^{i} \log _{2}\left(1+c_{5}^{i} \Gamma_{i}\right) \mathrm{bit} / \mathrm{s}
$$

where $c_{4}^{i}=1 / c_{3}^{i}$ and $c_{5}^{i}=-c_{2}^{i} / \ln \left(\mathrm{BER}_{i} / c_{1}^{i}\right)$. In Fig. 2, the approximation is compared with the expression in (3) at BER = $10^{-2}$ and BER $=10^{-5}$, respectively. It is shown that (5) is a good approximation for throughput versus SINR for a fixed BER.

In reality, the channel estimation errors can affect the performance of adaptive modulation. In this paper, the perfect channel estimation is assumed and it is used in many literature works. Many analyses for the effects of channel estimation errors on adaptive modulation can be found in [11], [13], and [14].

\section{B. Traditional Power Control}

In a traditional power control problem [5], the SINR of each user is maintained greater than or equal to some threshold $\gamma_{i}$ that can provide the adequate link quality. The problem is given by

$$
\begin{aligned}
& \min _{\gamma_{i}} \sum_{i=1}^{K} P_{i}(\boldsymbol{\Gamma}) \\
& \text { subject to } \Gamma_{i} \geq \gamma_{i}, \quad \forall i
\end{aligned}
$$

where $\boldsymbol{\Gamma}=\left[\Gamma_{1} \ldots \Gamma_{K}\right]^{T}$. In this kind of power control, a fixed and predefined targeted SINR threshold $\gamma_{i}$ for the desired modulation rate and BER is assigned to each user. Then, the transmitted powers are updated to ensure users' targeted SINRs

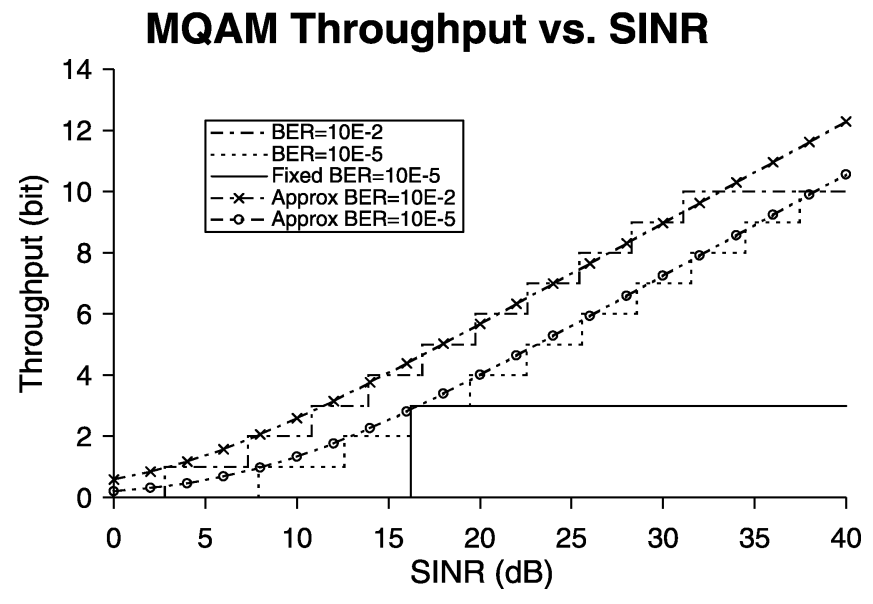

Fig. 2. BER approximation and BER standard formula for MQAM.

without considering their channel conditions. The system works perfectly in low SINR areas. When the targeted SINRs become high enough, the overall transmitted power will start to increase rapidly. If the targeted SINRs are larger than some specific values, there are no feasible solutions, i.e., the receivers cannot get enough SINR levels, no matter how large the transmitted powers are. One of the underlying reasons for such a problem is that the users with bad channel responses require too many transmitted powers; thus, they introduce unnecessarily high CCI to others. Therefore, having a fixed targeted SINR threshold is not an optimal power control approach.

\section{Optimization Problem}

All links are assumed to apply MQAM with throughput $T_{i}$ within a range $\left[T_{i}^{\min }, T_{i}^{\max }\right]$, according to their channel conditions, while the overall network throughput $T=\sum_{i=1}^{K} T_{i}$ is maintained greater than or equal to a constant $R$. $R$ is equal to the sum of the fixed targeted throughput in the previous scheme [5] in (6). $R$ should be selected such that the system is always 
feasible. If $R$ is too large, it is likely that the overall network throughput will be larger than the overall system capacity, as a result there will be no solution. Each time, the links with bad channel conditions sacrifice their throughput, i.e., they use lower SINR thresholds, which reduce the unnecessary CCI. The links with good channel conditions use higher SINR thresholds, i.e., more bits per symbol are selected, which increases the network throughput. For each link, the time-average throughput is a constant to ensure fairness, and the throughput is "water filled" at different times. For the whole system at any specific time, the overall network throughput is allocated to different links, according to their channel conditions so as to minimize the overall transmitted power. The value of $R$ is also equal to the sum of all users' time average throughput, so that the sum of users' time average throughput and the overall network throughput can coincide. This problem can be summarized as

$$
\begin{aligned}
& \min _{T_{i}, P_{i}} \sum_{i=1}^{K} P_{i} \\
& \text { subject to }\left\{\begin{array}{l}
\text { Feasibility : }(\mathbf{I}-\mathbf{D F}) \mathbf{P} \geq \mathbf{u}, \\
\text { Network Performance }: T \geq R, \\
\text { Throughput Range : } T_{i}^{\min } \leq T_{i} \leq T_{i}^{\max }, \\
\text { Fairness : } \lim _{N \rightarrow \infty} \frac{\sum_{n=1}^{N} T_{i}(n)}{N}=\text { const }_{i}
\end{array}\right.
\end{aligned}
$$

where $R=\sum_{i=1}^{K} E\left(T_{i}(n)\right)$. Only one type of users is assumed, so const ${ }_{i}=$ const $_{j}, \forall i, j$. The feasibility constraint $(\mathbf{I}-\mathbf{D F}) \mathbf{P} \geq \mathbf{u}$ is the matrix expression for the equalities $\Gamma_{i} \geq \gamma_{i}, \forall i[5]$, where $\mathbf{u}=\left[u_{1}, \ldots, u_{K}\right]^{T}$, $u_{i}=\gamma_{i} \mathbf{w}_{i}^{H} N_{i} \mathbf{w}_{i} /\left(\rho_{i i} G_{i i}\left\|\mathbf{w}_{i}^{H} \mathbf{h}_{i i}\right\|^{2}\right), \mathbf{P}=\left[P_{1}, \ldots, P_{K}\right]^{T}$, $\mathbf{D}=\operatorname{diag}\left\{\gamma_{1}, \ldots, \gamma_{K}\right\}$, and

$$
\left[\mathbf{F}_{i j}\right]= \begin{cases}0, & \text { if } j=i, \\ \frac{\rho_{j i} G_{j i}\left\|\mathbf{w}_{i}^{H} \mathbf{h}_{j i}\right\|^{2}}{\rho_{i i} G_{i i}\left\|\mathbf{w}_{i}^{H} \mathbf{h}_{i i}\right\|^{2}}, & \text { if } j \neq i .\end{cases}
$$

In the problem defined above, the complexity lies in the optimization over time and grows rapidly with the number of users. In Section III, algorithms are developed to reduce the complexity and distribute the computing efforts to both the system level and the user level.

\section{Problem PARtition AND AdAPtive Algorithms}

\section{A. Problem Partition}

The difficulties of solving (7) lie in the feasibility and fairness constraints. First, in the feasibility constraint, if the users' transmitted powers are fixed, the targeted SINR $\gamma_{i}$ is linearly constrained. On the other hand, if $\gamma_{i}$ is fixed, the constraint is linear for $\mathbf{P}$. However, if both SINRs and powers are considered, it is a bilinear matrix inequality (BMI) problem [32]. The BMI problem is nonconvex and nonlinear. Only limited tools are available in the literature to find the solutions [32]. Second, in the fairness constraint, the throughput is considered at the different times. It is very difficult to solve the problem by traditional dynamic programming because the distributions of the received SINRs and transmitted powers are extremely hard to model and calculate. Therefore, the problem defined in (7) is too difficult to find an analytically optimal solution. A heuristic way is needed to obtain a suboptimal solution with relatively good performances.

If the fairness constraint is not considered, the problem in (7) is a pure constrained optimization problem. With the consideration of fairness, the motivation to solve the problem comes from jointly considering the throughput ranges and fairness constraints. First, the users report the ranges of throughput that they can accept. Then, the system decides how to allocate the throughput to each user each time, according to these ranges. The acceptable throughput ranges are modified by the users' transmission history. Each time, some users may have more throughput, while others have less. Then, the users with more throughput will become less aggressive about transmitting and will request smaller throughput ranges in the near future, and vice versa. From the above idea, the optimization problem in (7) is divided into two subproblems.

1) At the user level, in order to ensure fairness, the users trace their histories of throughput and report the ranges of throughput that they can accept to the system at current time.

2) At the system level, for the whole network each time, the system determines the optimal throughput allocation to different users, and this allocation requires the lowest overall transmitted power.

Therefore, the overall transmitted power is minimized each time, and fairness is guaranteed. However, the optimal solution for (7) is not guaranteed to be achieved. But, from the simulation results in Section IV, the significant performance improvements over the traditional system [5] will be shown.

An illustrative example for two users is shown in Fig. 3. The two axes represent the two users' desired SINRs that are related to their throughput. The provided ranges are the required SINRs for the throughput ranges that the users provide, and these ranges are also restricted by the feasibility constraint. On the dashed line, the overall network throughput $T=T_{1}+T_{2}$ is a constant. At the system level, the goal is to find what is the optimal point each time that requires the minimum overall transmitted power, within the range (shown as the polyhedra) and under the overall throughput constraint $T \geq R$. At the user level, the problem is how to change the throughput ranges over different times to ensure fairness. For example, if user 1 is assigned to have small throughput now, he will be more aggressive about transmitting his data in the future. Consequently, the throughput range will move to the right within the practical range, and user 1 has to be assigned the higher throughput in the future.

\section{B. Adaptive Algorithm for Throughput Range at the User Level}

In this section, the first subproblem will be solved. An adaptive algorithm is developed at the user level to report the acceptable throughput ranges back to system, to ensure fairness. The key idea is to adapt the throughput ranges with joint consideration of the fairness constraint. Instead of having a fixed throughput range $\left[T_{i}^{\min }, T_{i}^{\max }\right]$ for each link, the throughput ranges are adaptively changed by taking into account the links' throughput histories. Assume the $i$ th link can select throughput $T_{i}^{\min }(n) \leq T_{i}(n) \leq T_{i}^{\max }(n)$ at time $n$, and the average desired throughput for the $i$ th link is $T_{i}^{\text {ave }}$. Each time, $T_{i}^{\min }(n+1)$ 


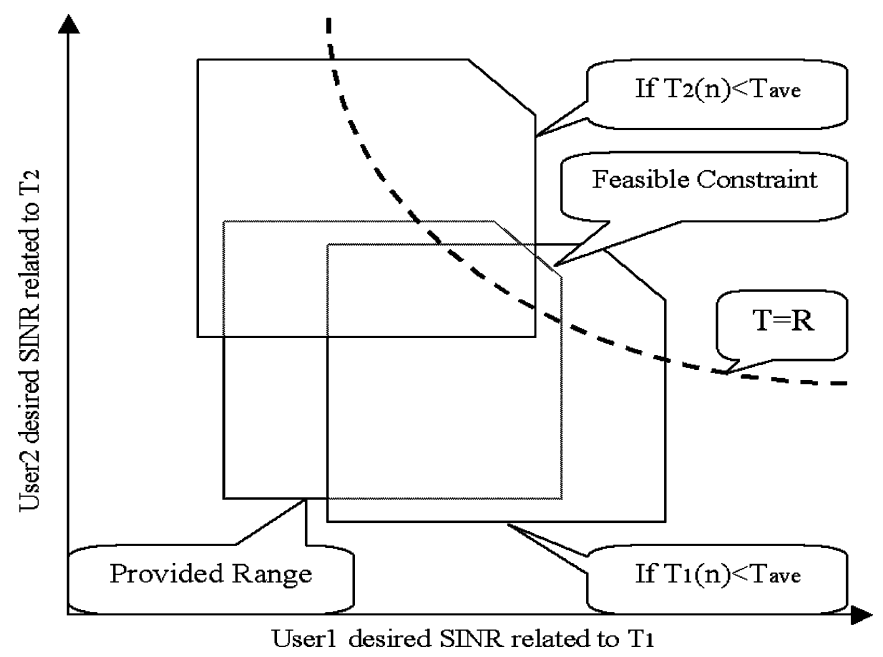

Fig. 3. Two-user example for problem partition.

and $T_{i}^{\max }(n+1)$ are modified by the current $T_{i}(n)$. When $T_{i}(n)$ is smaller than $T_{i}^{\text {ave }}, T_{i}^{\min }(n+1)$ and $T_{i}^{\max }(n+1)$ are increased so that there is a higher probability that the future throughput $T_{i}(n+1)$ is larger than $T_{i}^{\text {ave }}$. When $T_{i}(n)$ is larger than $T_{i}^{\text {ave }}$, $T_{i}^{\min }(n+1)$ and $T_{i}^{\max }(n+1)$ are decreased so that there is a higher probability that $T_{i}(n+1)$ is smaller than $T_{i}^{\text {ave }} \cdot T_{i}^{\min }(n+$ 1) and $T_{i}^{\max }(n+1)$ are bounded by $\hat{T}_{i}^{\min }$ and $\hat{T}_{i}^{\max }$, which are the practical minimum and maximum throughput boundaries that the $i$ th link can select, respectively. Their values are fixed and predefined by the system. In order to track the history of $T_{i}$, $T_{i}^{\text {mid }}(n)=T_{i}^{\operatorname{mid}}(n-1)+\beta\left(T_{i}(n)-T_{i}^{\text {ave }}\right), 0<\beta<1$, where $\beta$ is a constant that depends on how much delay the user can suffer. If the delay constraint is tight, $\beta$ should be selected as a relatively larger number so that the throughput range will move quickly to compensate the user's throughput loss at a specific time. If the user can suffer longer delay, $\beta$ could be selected as a relatively smaller number so that the user can wait until the channel becomes better to be compensated back. The selected value of $\beta$ is also affected by how rapidly the channels change. If the channels change slowly, a smaller $\beta$ is preferred, so that the user can wait; otherwise, a larger $\beta$ is selected. Each time, the throughput window is updated by

$$
\left\{\begin{array}{c}
T_{i}^{\mathrm{mid}}(n)=T_{i}^{\mathrm{mid}}(n-1)+\beta\left(T_{i}(n)-T_{i}^{\mathrm{ave}}\right) \\
T_{i}^{\min }(n+1)=\min \left(\operatorname { m a x } \left(T_{i}^{\mathrm{ave}}-T_{i}^{\mathrm{mid}}(n)\right.\right. \\
\left.\left.+\hat{T}_{i}^{\min }, \hat{T}_{i}^{\min }\right), \hat{T}_{i}^{\max }\right) \\
T_{i}^{\max }(n+1)=\max \left(\operatorname { m i n } \left(\hat{T}_{i}^{\max }-T_{i}^{\operatorname{mid}}(n)\right.\right. \\
\left.\left.+T_{i}^{\text {ave }}, \hat{T}_{i}^{\max }\right), \hat{T}_{i}^{\min }\right) .
\end{array}\right.
$$

The above throughput window may move to the opposite direction of the channel changing trend. When the channel is bad, the user selects less throughput. But in the next time, the user has to select a larger throughput because the throughput window moves to a higher throughput area, even if the channel is still bad. With the consideration of the channel changes, a scheme is developed so that the throughput window follows the channel changing trend. This problem can be categorized as a dynamic programming problem given by

$$
\begin{aligned}
& {\left[T_{i}^{\min }(n+1), T_{i}^{\max }(n+1)\right]=f_{n}\left(\left[T_{i}^{\min }(n),\right.\right.} \\
& \left.\left.T_{i}^{\max }(n)\right], v_{n}, T_{i}(n)\right), \quad n=0,1, \ldots, N-1
\end{aligned}
$$

where $f_{n}$ is a function to select the throughput window at time $n$, and $v_{n}$ is the control policy that has a different impact on the outcomes of $f_{n}$. The problem in (9) is extremely difficult to solve, but an intuitive idea can be applied to find a much simpler solution. Because $\beta$ may not be an integer, the throughput window developed in (8) may not be discrete. If the $i$ th user's assigned throughput at the current time $n$ is smaller than the median of all the users' assigned throughput in the adjacent cells, this means that the $i$ th user is possibly still under the bad channel condition. The lower throughput window is assigned to follow the channel condition, by using the floor of the original throughput window. Here, the floor is a function that finds the maximum integer immediately less than the real value. On the other hand, if the $i$ th user's throughput is larger than the median of the users' throughput among the adjacent cells, the higher throughput window is assigned to follow the channel condition by using the ceiling of the original throughput window. Here, the ceiling is a function that finds the minimum integer immediately greater than the real value.

In addition, when a user is trapped in a bad channel for a long time, instead of assigning him with a very high throughput range, the algorithm should be able to assign this user with lower throughput. By doing so, the user will not cause too much CCI to others, and the system performance can be improved. The history of $T_{i}^{\min }(n)$ is tracked. If the user detects $Z$ consecutive $T_{i}^{\min }(n)$ equal to $\hat{T}_{i}^{\max }$, the user will report the acceptable throughput range as $\left[\hat{T}_{i}^{\min }, T_{i}^{\max }(n)\right]$, instead of $\left[T_{i}^{\min }(n), T_{i}^{\max }(n)\right]$. Consequently, the system is able to assign the minimal throughput to the user. The throughput ranges are updated by users to BS every power update interval. Because the ranges are discrete and limited by the hardware, the associated overheads to report these ranges are small. In a real system, this information is coded by a powerful error control code to ensure that it comes through without errors. In each iteration, users' throughput windows are updated by the following algorithm.

$$
\begin{aligned}
& \text { Adaptive Algorithm for Each User's } \\
& \text { Throughput Window } \\
& \text { 1) Initialization: } T_{i}^{\min }(0)=\hat{T}_{i}^{\min } \text {, } \\
& T_{i}^{\max }(0)=\hat{T}_{i}^{\max }, T_{i}^{\operatorname{mid}}(0)=T_{i}^{\text {ave }} \\
& \text { 2) Iteration: } \\
& T_{i}^{\mathrm{mid}}(n)=T_{i}^{\mathrm{mid}}(n-1)+\beta\left(T_{i}(n)-T_{i}^{\mathrm{ave}}\right) ; \\
& \text { if } T_{i}(n)>\operatorname{median}\left(T_{j}(n)\right), j \in \\
& \text { all adjacent CCI cells, } \\
& T_{i}^{\min }(n+1)=f \operatorname{lloor}\left(\operatorname { m i n } \left(\operatorname { m a x } \left(T_{i}^{\text {ave }}-\right.\right.\right. \\
& \left.\left.\left.T_{i}^{\operatorname{mid}}(n)+\hat{T}_{i}^{\min }, \hat{T}_{i}^{\min }\right), \hat{T}_{i}^{\max }\right)\right) ; \\
& T_{i}^{\max }(n+1) \stackrel{\text { floor }}{=} \max \left(\operatorname { m i n } \left(\hat{T}_{i}^{\max }-\right.\right. \\
& \left.\left.\left.T_{i}^{\operatorname{mid}}(n)+T_{i}^{\text {ave }}, \hat{T}_{i}^{\max }\right), \hat{T}_{i}^{\min }\right)\right) \text {; } \\
& \text { else } \\
& T_{i}^{\min }(n+1) \quad=\quad \operatorname{ceiling}\left(\operatorname { m i n } \left(\operatorname { m a x } \left(T_{i}^{\text {ave }}-\right.\right.\right. \\
& \left.\left.\left.T_{i}^{\operatorname{mid}}(n)+\hat{T}_{i}^{\min }, \hat{T}_{i}^{\min }\right), \hat{T}_{i}^{\max }\right)\right) \text {; }
\end{aligned}
$$




$$
\begin{aligned}
& T_{i}^{\max }(n+1)=\operatorname{ceiling}\left(\operatorname { m a x } \left(\operatorname { m i n } \left(\hat{T}_{i}^{\max }-\right.\right.\right. \\
& \left.\left.\left.T_{i}^{\operatorname{mid}}(n)+T_{i}^{\text {ave }}, \hat{T}_{i}^{\max }\right), \hat{\hat{T}}_{i}^{\min }\right)\right) ; \\
& \text { 3) Feedback the Acceptable Throughput } \\
& \text { Ranges to BS: } \\
& \quad \text { if } T_{i}^{\min }(n+1)=T_{i}^{\min }(n)=\ldots=T_{i}^{\min }(n-Z+ \\
& \text { 1)= } \hat{T}_{i}^{\max }, \text { report }\left[\hat{T}_{i}^{\min }, T_{i}^{\max }(n)\right] ; \\
& \quad \text { else, report }\left[T_{i}^{\min }(n), T_{i}^{\max }(n)\right] .
\end{aligned}
$$

If a user is never trapped in the bad channel for a long period of time, when $T_{i}(n)$ is continuously less than $T_{i}^{\text {ave }}$ for some time, $T_{i}^{\min }(n)$ is increased to $T_{i}^{\text {ave }}$. Then, the next $T_{i}(n+1)$ has to select the throughput equal to or greater than $T_{i}^{\text {ave }}$; consequently, $T_{i}^{\text {mid }}(n)$ stops increasing. The same analysis can be applied to $T_{i}^{\max }(n)$. Since $T_{i}^{\min }(n)$ and $T_{i}^{\max }(n)$ are bounded and are linearly modified by $T_{i}^{\text {mid }}(n), T_{i}^{\text {mid }}(n)$ is also bounded. If $T_{i}^{\text {mid }}(n)$ is rearranged and summed over the different times

$$
\frac{\sum_{n=1}^{N} T_{i}(n)}{N}=T_{i}^{\mathrm{ave}}+\frac{\left(T_{i}^{\mathrm{mid}}(N)-T_{i}^{\mathrm{ave}}\right)}{\beta N} .
$$

The second term on the right-hand side decreases to zero as $N \rightarrow \infty$. So, $\lim _{N \rightarrow \infty}\left(\sum_{n=1}^{N} T_{i}(n) / N\right)=T_{i}^{\text {ave }}$, i.e., the system is fair, so that each user's time-average throughput is a constant.

If a user is trapped in the bad channel for a long period of time and detects $Z$ consecutive $T_{i}^{\min }(n)$ equal to $\hat{T}_{i}^{\max }$, the user will report the acceptable throughput range as $\left[\hat{T}_{i}^{\min }, T_{i}^{\max }(n)\right]$. Under this condition, $T_{i}^{\text {mid }}(n)$ will not be bounded. If the channel becomes better in the future and the system assigns more throughput to this user, $T_{i}^{\operatorname{mid}}(n)$ will be increased. Consequently, $T_{i}^{\max }(n)$ will be less than $\hat{T}_{i}^{\max }$, and the second term on the right-hand side of (10) will approach to zero, asymptotically. If a user is trapped in the bad channel indefinitely, $T_{i}^{\text {mid }}(n)$ will go to negative infinity, and fairness constraint cannot be satisfied. In practice, this situation seldom happens. If it does happen, there is no practical meaning to guarantee fairness for this user because this user will cause too much CCI that will reduce the system performance a lot.

\section{Adaptive Algorithms for Throughput Allocation at the System Level}

In this section, the second subproblem will be solved, and three adaptive algorithms will be developed at the system level to allocate throughput to different users each time to generate the minimum overall transmitted power. The first one is a full search algorithm that can guarantee to find the optimal solution each time, but the complexity is very high. The second one is a fast search algorithm that analyzes which users contribute more to the overall transmitted power. The last one is an adaptive algorithm by assuming that the throughput is continuous and approximated by (5). Then, the throughput allocation result is projected to the closest discrete value that satisfies all the constraints.

Full Search Algorithm: Because there is only a limited number of discrete throughput $T_{i}$ that each user can select, and there are only limited number of users, a full search method can be applied to find the optimal throughput allocation. The users provide the acceptable throughput ranges to the system. The system calculates the overall transmitted powers of all combinations of $T_{i}$ by the iterative algorithm under the constraints in (7). The throughput allocation that generates the lowest overall transmitted power is selected. The adaptive algorithm can find the optimal solution each time, but it has very high complexity. The complexity is increased exponentially with the number of users, which is not acceptable in practice. It can be used as a performance bound. The full search adaptive algorithm is given by the following.

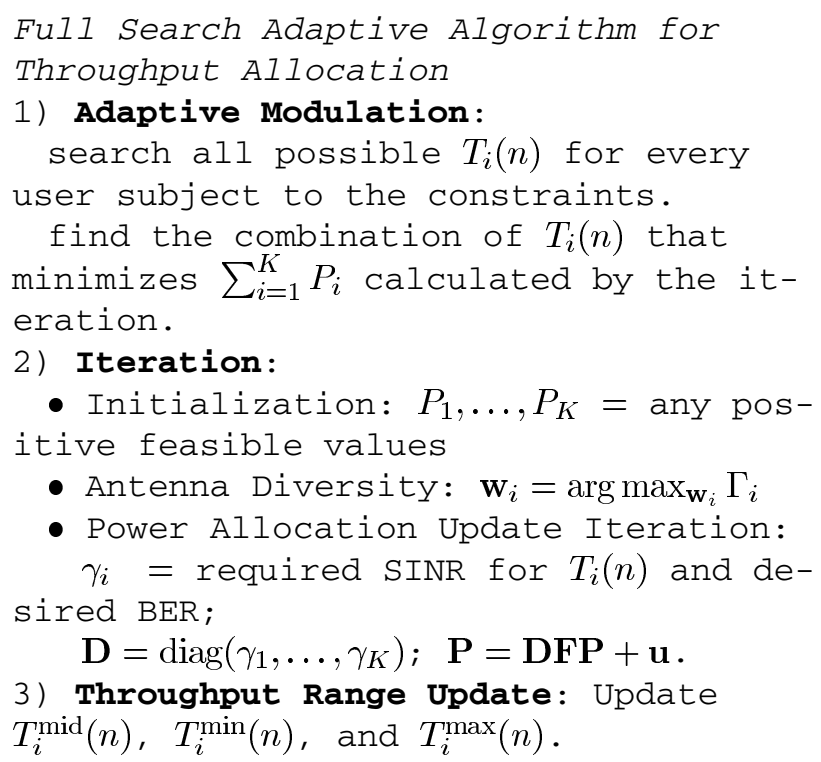

Fast Search Algorithm: In order to reduce complexity, a fast search algorithm is developed. The system needs to find out which users contribute more to the overall transmitted power. The gradient of overall transmitted power to each user's targeted SINR is derived. If the users with larger gradients can sacrifice their SINRs a little bit, the overall transmitted power will be reduced significantly.

In the Perron-Frobenius theorem [34], if the spectrum radius of DF, $\rho(\mathbf{D F})$, i.e., the maximum absolute eigenvalue, is less than one, the minimum overall transmitted power is achieved when $\Gamma_{i}=\gamma_{i}, \forall i$, and $P_{\text {sum }}$ can be written as

$$
P_{\text {sum }}=\sum_{i=1}^{K} P_{i}\left(T_{i}(n)\right)=\mathbf{1}^{T}(\mathbf{I}-\mathbf{D F})^{-1} \mathbf{u}
$$

where $\mathbf{1}=[1 \ldots 1]^{T}$. Define $\mathbf{Q}=[\mathbf{I}-\mathbf{D F}]^{-1}$. If $(\mathbf{D F}) \in$ $R^{K \times K}$ and $\rho(\mathbf{D F})<1$, then $\mathbf{Q}=\sum_{j=0}^{\infty}(\mathbf{D F})^{j}$. Since $\mathbf{D}=$ $\operatorname{diag}\left(\gamma_{1}, \ldots, \gamma_{K}\right)$, and $[\mathbf{F}]_{i j}>0, \forall i, j$, if $\gamma_{j}, j=1 \ldots K$, $j \neq i$ is fixed, every component in $\mathrm{Q}$ is a function of $\left(\gamma_{i}\right)^{j}, j=$ $1 \ldots \infty$ with nonnegative coefficients. In vector $\mathbf{u}$, every $u_{i}$ has the nonnegative coefficients as well. So, $P_{\text {sum }}=\sum_{i=1}^{K} P_{i}=$ $\mathbf{1}^{T} \mathbf{Q}^{-1} \mathbf{u}$ is also a function of $\left(\gamma_{i}\right)^{j}$ with nonnegative coefficients. The only situation where the coefficients are zeros is when the antenna diversity uses a null for the desired mobile user. This hardly happens in practice. Since $\gamma_{i}>0, \forall i$, when the other $\gamma_{j}, j=1 \ldots K, j \neq i$ is fixed, $P_{\text {sum }}$ is a convex and increasing function of $\gamma_{i}$. From (5), $\gamma_{i}$ is an increasing and convex function of throughput $T_{i}$. So, $P_{\text {sum }}$ is also an increasing and convex function of $T_{i}$, when the other $T_{j}, j=1 \ldots K, j \neq i$ is 
fixed. Consequently, the overall transmitted power is minimized when the network throughput constraint is equal, i.e., $T=R$. This is because any $T_{i}$ can be reduced to have smaller overall transmitted power, if $T>R$.

Now, the gradients of overall transmitted power can be deduced. If the derivatives are taken with respect to $\gamma_{i}$ at both sides of (11), the $i$ th element of gradient $\mathrm{g}=\left[g_{1} \ldots g_{K}\right]^{T}$ is given by [21]

$$
g_{i}=\frac{\partial P_{\mathrm{sum}}}{\partial \gamma_{i}}=\frac{c_{i} P_{i}}{\Gamma_{i}}
$$

where $c_{i}=\mathbf{1}^{T}(\mathbf{I}-\mathbf{D F})^{-1} \mathbf{v}_{i}$, and $\left[\mathbf{v}_{i}\right]_{j}=1$, if $j=i ;\left[\mathbf{v}_{i}\right]_{j}=$ 0 , otherwise. The value of $c_{i}$ reflects how severe the CCI is. When the CCI is large, $c_{i}$ tells how much the $i$ th user causes the CCI to other users. When the CCI is small, $c_{i} \approx c_{j}, \forall i$, $j$. Since the adaptive algorithm only needs the direction of the gradients and does not need the amplitudes, the value of $c_{i}$ can be ignored, i.e., $c_{i}=1, \forall i$, when the CCI is small. Equation (12) is very significant in that it provides a very simple way to find the gradients. In this case, SINRs can be measured at each base station's antenna diversity output, and the feedback channels can be used to get the mobile transmitted power values to calculate the gradients. Consequently, the complexity can be reduced greatly.

With the gradients, a greedy algorithm is developed. First, because the network throughput constraint $T=R$ is nonlinear, the overall transmitted power $P_{\text {sum }}$ is no longer a convex function of $\gamma_{i}$ under this constraint. The gradients of different users are compared. If a user with a larger gradient selects lower throughput, i.e., he requires a lower targeted SINR threshold, the overall transmitted power is greatly reduced. So, first the throughput that generates the lowest overall transmitted power is decided for the user with the highest gradient, subject to the constraints. When the throughput of the user with the largest gradient is changed, the throughput of the other users is modified in the order from the lower gradient to higher gradient to compensate the network throughput constraint $T=R$. By doing this, more throughput is allocated to the users with small gradients, and less throughput is assigned to the users with large gradients; consequently, the overall transmitted power will be reduced significantly. Note that the throughput of the user with the largest gradient may not end up with the lowest throughput $T_{i}^{\min }(n)$ because the increase of the sum of other user's powers may be larger than the decrease of this user's power. In the next iteration, the throughput of the user with the largest gradient is fixed, and the system finds the optimal throughput for the user with the second highest gradient, and so on, until we find the throughput of the last user in the row. Because every user only searches for a fixed amount of throughput range and reordering is needed, if the gradient is calculated by (12) and $c_{i} \approx c_{j}, \forall i, j$ for simplicity, this suboptimal algorithm has the complexity of only $O\left(K^{2} \log _{2} K\right)$. If the CCI is severe and $c_{i} \neq c_{j}$, then the complexity is $O\left(K^{3} \log _{2} K\right)$. If the user index is rearranged from the largest gradient to the lowest, i.e., $g_{1} \geq g_{2} \geq \ldots \geq g_{K}$, and any nonfeasible solution has $P_{\text {sum }}=\infty$, the suboptimal adaptive iterative algorithm is summarized by the following.
Fast Search Adaptive Algorithm for

Throughput Allocation

1) Initialization: $T_{1}(0)=T_{1}^{\text {ave }}, \ldots, T_{K}(0)=$ $T_{1}^{\text {ave }}, P_{1}, \ldots, P_{K}=$ any feasible positive const.

2) Adaptive Modulation

for $i=1$ to $K$

for $T_{i}=T_{i}^{\min }(n)$ to $T_{i}^{\max }(n)$

1) Modify from $T_{K}(n)$ to $T_{i+1}(n)$ to

satisfy the constraint $T=R$ (exhaust

$T_{K}$ first.)

2) Run iteration

- Antenna Diversity: $\mathbf{w}_{i}=$

$\arg \max _{\mathbf{w}_{i}} \Gamma_{i}$

- Power Allocation Update Itera-

tion:

$\gamma_{i}=$ required SINR for $T_{i}$ and

desired BER, $\mathbf{D}=\operatorname{diag}\left(\gamma_{1} \ldots \gamma_{K}\right), \mathbf{P}=$ $\mathrm{DFP}+\mathbf{u}$.

3) Find $T_{i}$ that generates the

lowest power for the $i$ th user.

end

end

3 ) Throughput Range Update: Update $T_{i}^{\operatorname{mid}}(n), T_{i}^{\min }(n)$, and $T_{i}^{\max }(n)$.

The algorithm is suboptimal because the optimal throughput for one user may not be optimal for all users. The algorithm may stop at some local minimum points or the boundary points. From the simulation results that will be shown, the suboptimal algorithm has relatively good performance.

Projected Gradient Algorithm: As we have stated, the feasible constraint in (7) is a BMI constraint. Here, the approximation of throughput in (5) is used, and a projected gradient algorithm [32] is developed to change each user's targeted SINR to find the minimal overall transmitted power. The throughput allocation results are probably not integers. The results are projected to the nearest discrete throughput allocation that satisfies the constraints. Then, the above two steps are employed again, until the discrete throughput allocations are the same in two consecutive iterations.

First, the projected gradient method will be developed. The throughput is now supposed to be continuous and has the value $\tilde{T}_{i}$. If each user's targeted SINR is changed by the gradient in (12), the overall throughput constraint $\sum_{i=1}^{K} \tilde{T}_{i}=R$ cannot be satisfied. The gradient needs to be modified such that the overall throughput constraint holds. The plane that is tangent to the curve $\sum_{i=1}^{K} \tilde{T}_{i}=R$ at point $\left[\gamma_{1}, \ldots, \gamma_{K}\right]$ needs to be found, where $\gamma_{i}=\left(2^{\tilde{T}_{i} / c_{4}^{i}}-1\right) / c_{5}^{i}$. Without loss of generality, this plane can be moved to the origin. The plane can be expressed as: $\sum_{i=1}^{K} k_{i} x_{i}=0$, where $k_{i}=c_{4}^{i} c_{5}^{i} /\left(1+c_{5}^{i} \gamma_{i}\right)$. The modified gradient is given by $\mathbf{q}=\left[q_{1} \ldots q_{K}\right]^{T}$. By the definition of projection, vector $\mathbf{q}$ satisfies equation $\|\mathbf{g}-\mathbf{q}\|^{2}=$ $\min _{\forall \mathbf{q} \in \text { plane }}\|\mathbf{g}-\mathbf{q}\|^{2}$. The right-hand side needs to be minimized to get the optimal vector, i.e., the projection $\mathbf{q}$.

The best throughput allocation $\tilde{T}_{i}$ is obtained from the above projected gradient algorithm. $\tilde{T}_{i}$ needs to be projected to a dis- 

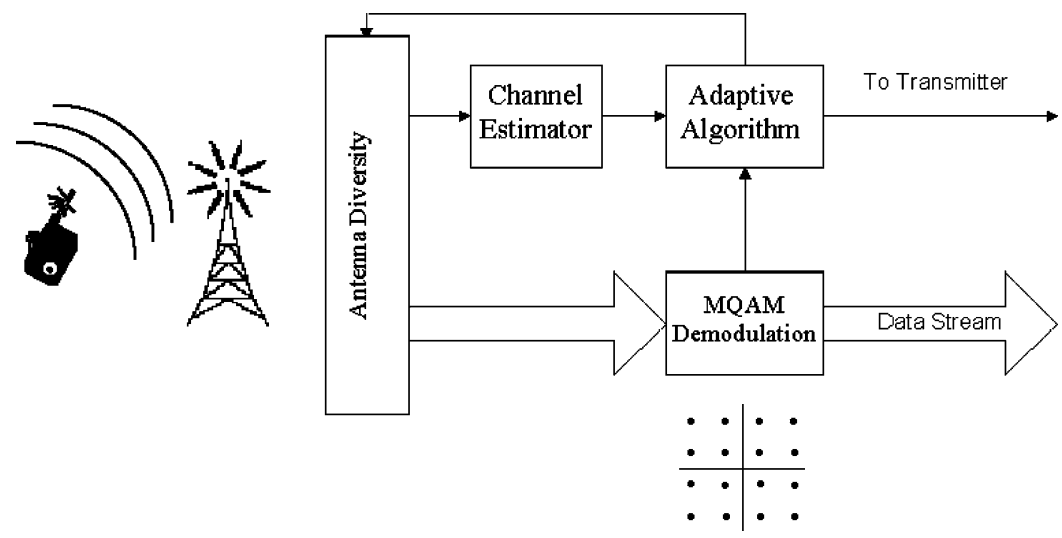

Fig. 4. Power control and throughput management system.

crete value. The projection problem can be written as

$$
\begin{aligned}
& \min _{T_{i}}\left\|\tilde{T}_{i}-T_{i}\right\|^{2} \\
& \text { subject to } \sum_{i=1}^{K} T_{i}=R, T_{i}^{\min }(n) \leq T_{i} \leq T_{i}^{\max }(n), \\
& \quad \text { and }(\mathbf{I}-\mathbf{D F}) \mathbf{P} \geq \mathbf{u}
\end{aligned}
$$

where $\tilde{T}_{i}$ is projected to the discrete value with the constraint $\sum_{i=1}^{K} T_{i}=R$. However, the discrete throughput projection may not be feasible or not in the ranges. If so, the second closest point needs to be found to see if it satisfies all the constraints. The search is continued until a feasible solution is found. The projected gradient algorithm is given by the following.

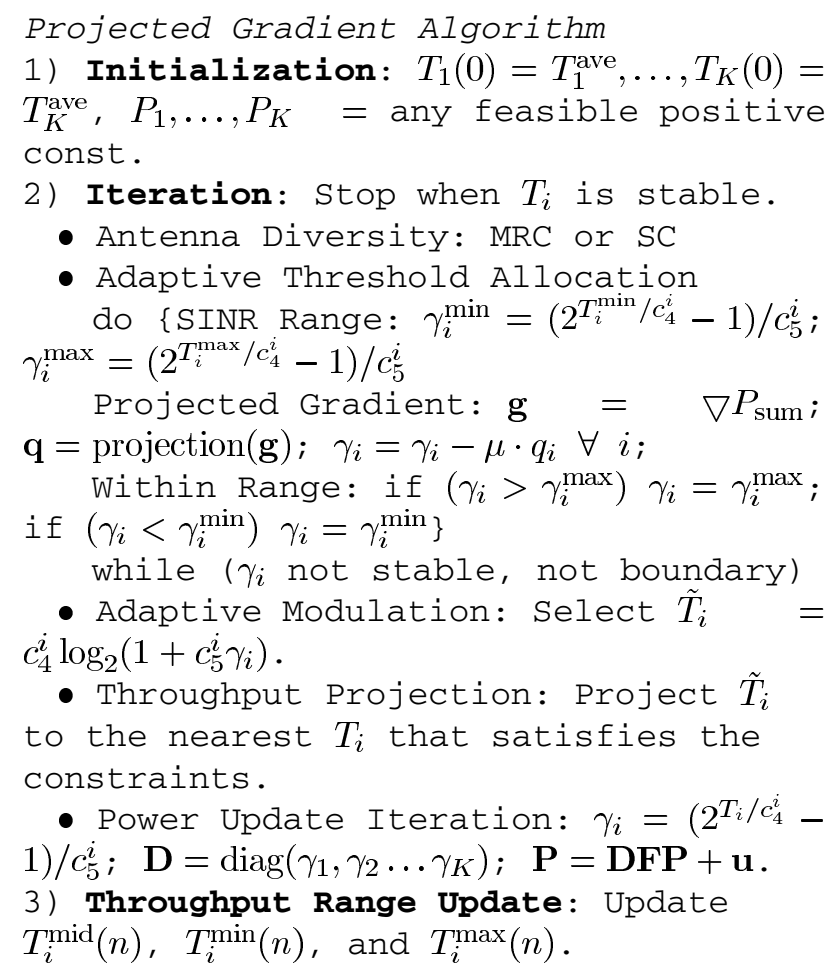

In the algorithm, $\mu$ is a small constant, whose value decides the rate of convergence and the variance of the final results. Whether or not $\gamma_{i}$ is stable is decided by comparing the maximum difference of $\gamma_{i}$ in two consecutive steps. The algorithm has complexity of $O\left(K^{3}\right)$. However, because two iterations are needed each time, the complexity is higher than that of the fast search algorithm but still much lower than that of the full search algorithm, when the number of users is large. From the simulation results, it will be shown that the projected gradient algorithm can find the optimal solution each time.

The algorithm starts from any feasible rate and power allocation. In each iteration, the gradient of the overall transmitted power is calculated and projected on a plane where the network performance constraint is satisfied. This modified gradient is at least pointing in the direction where the overall transmitted power is increasing. The algorithm modifies the SINR allocation at the opposite direction of this modified gradient so that the new overall transmitted power is less than or equal to that of the old iteration. When the algorithm finds the SINR allocation solution, this SINR allocation must be feasible, and the transmitted powers are updated by fixing the targeted SINRs. This power update iteration converges to a unique solution [2], [5].

\section{Joint Power Control and Throughput Management System}

With the adaptive algorithms, a joint power control and throughput management system is constructed in Fig. 4. Because of users' multipath fading, shadowing, and random locations in their respective cells, the channel conditions are varying. Therefore, accurate techniques for "real time" estimations of channel conditions are essential [11], [14], [31]. The fluctuations of channels are assumed to be tracked perfectly by the base stations. This information is sent back to the mobile users via an error-free feedback channel. The time delay in this feedback channel is also assumed to be negligible, compared to the speed of channel and CCI variations. All these assumptions are reasonable in slowly varying channels.

The way the system works and the distribution of computing efforts are shown as follows. At the user level, the users compute and provide the system with their acceptable throughput ranges, according to their transmission histories and current channel conditions. At the system level, where the base stations have much stronger computing power, the adaptive algorithm module gets the estimation of users' channel responses from the channel estimation module. Then, power control and modulation rates are computed. The power control and 
TABLE I

NORMALIZED TRANSMITTED POWER With RESPECT to NUMBER OF ANTENNAS

\begin{tabular}{c|c|c|c|c|c|c}
\hline No. of antennas & 2 & 3 & 4 & 5 & 6 & 7 \\
\hline MRC $\left(B E R=10^{-2}\right)$ & 0.2722 & 0.1271 & 0.0904 & 0.0598 & 0.0468 & 0.0412 \\
\hline $\mathrm{SC}\left(\mathrm{BER}=10^{-2}\right)$ & 0.3746 & 0.2095 & 0.1677 & 0.1260 & 0.1083 & 0.1065 \\
\hline $\mathrm{MRC}\left(\mathrm{BER}=10^{-5}\right)$ & 0.1519 & 0.0787 & 0.0572 & 0.0463 & 0.0349 & 0.0264 \\
\hline $\mathrm{SC}\left(\mathrm{BER}=10^{-5}\right)$ & 0.1958 & 0.1248 & 0.0873 & 0.0797 & 0.0716 & 0.0655 \\
\hline
\end{tabular}
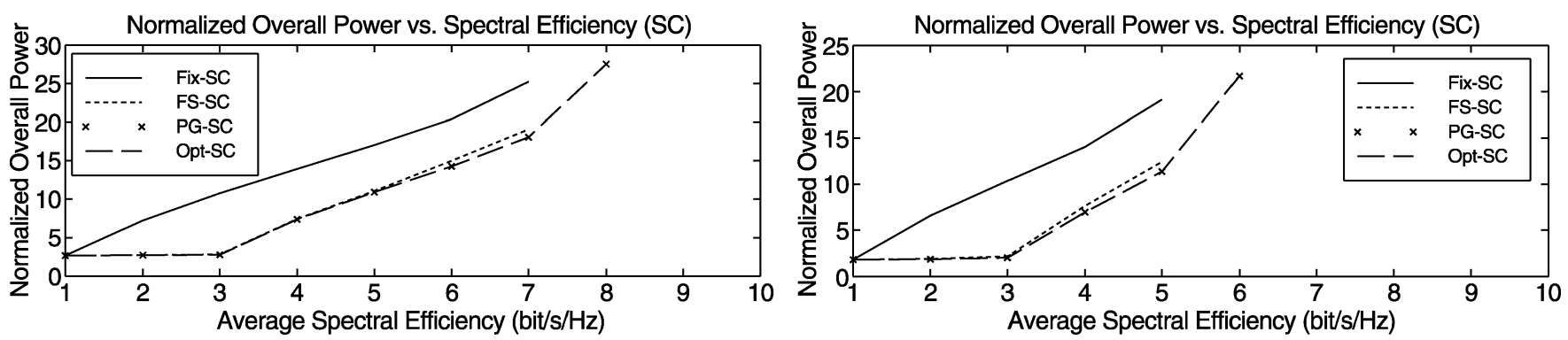

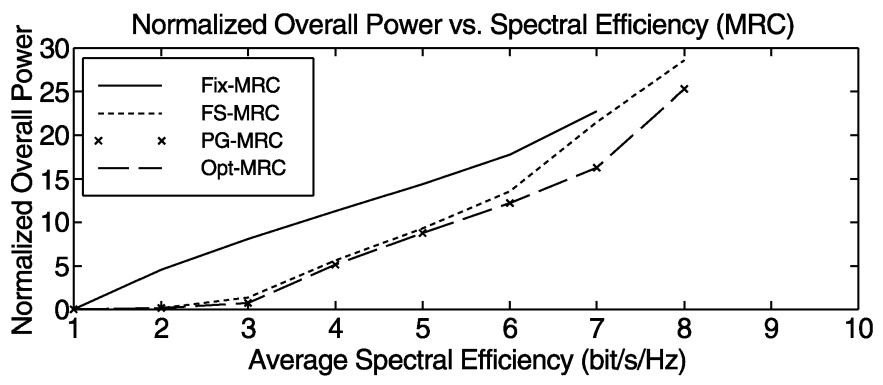

(a)

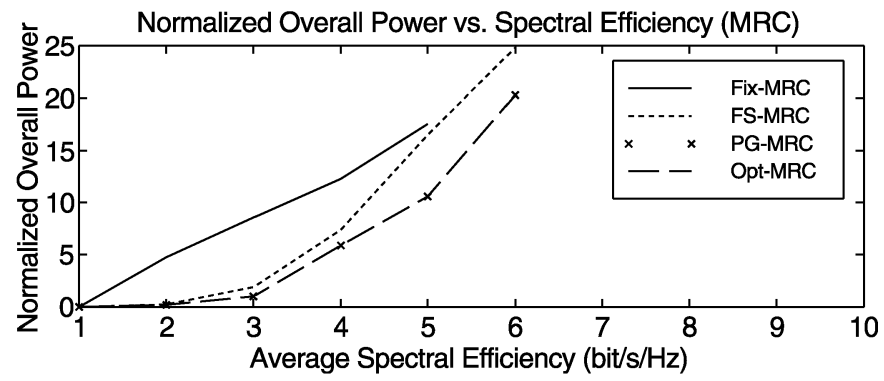

(b)

Fig. 5. Normalized power (in decibels) versus throughput. (a) $B E R=10^{-2}$. (b) $B E R=10^{-5}$.

best throughput allocation information is sent back to the mobile users. Then the mobile users, accordingly, adapt their transmission rates and powers.

For the mobile device with battery power supply, the maximum transmitted power is limited. In the optimization problem, the maximum power constraint can also be considered. In the proposed approaches, this constraint can be easily implemented by the full search and fast search algorithms. The algorithms are modified such that only the throughput allocation that satisfies the maximum power constraint will be selected. However, in the projected gradient method, the maximum power constraint will impose another very complex and nonlinear constraint in the proposed adaptive algorithm.

\section{Simulation Results}

In order to evaluate the performances of the proposed algorithms, a network with hexagonal cells is simulated. The radius of each cell is $1000 \mathrm{~m}$. Two adjacent cells do not share the same channel, i.e., the reuse factor is 7 . There are 50 cells in the networks. One base station is placed at the center of each cell. In each cell, one user is placed randomly with a uniform distribution. In the simulation, the fading is considered as complex Gaussian distributions with three multipaths of equal powers. The fading is independent between two resource allocation intervals. Each base station has a $P$-element antenna array. Noise power is $10^{-3} . Z=50$. A 3-dB log-normal distribution is considered. In this paper, the two slopes path loss model
[33] is applied to obtain the average received power as a function of distance. We select the basic path loss exponent as 2, the additional path loss exponent as 2 , the base station antenna height as $50 \mathrm{~m}$, the mobile antenna height as $2 \mathrm{~m}$, and the carrier frequency as $900 \mathrm{MHz}$. In Table I, the overall transmitted power of our proposed system is shown with respect to a different number of antennas. The value is normalized with the case where only single antenna is applied. Two different BER requirements $\left(\mathrm{BER}=10^{-2}\right.$ and $\mathrm{BER}=10^{-5}$ ) are shown, respectively. The overall transmitted power can have a reduction of about $75 \%$ to $95 \%$ for MRC compared to the single antenna case. The performance of SC is consistently worse than that of MRC. Since SC can apply noncoherent modulation, the complexity is much smaller. When the desired BER is decreased, SC performs closer to MRC. With the number of antennas $P$ increasing, from simulations, the decrease of powers saturates around $P=4$. Therefore, $P=4$ is chosen for rest of simulations.

In Fig. 5(a) and (b), the normalized overall transmitted power as a function of average spectral efficiency is compared for the fixed scheme [5], fast search scheme, projected gradient algorithm, and optimal full search scheme with MRC and SC diversity at $\mathrm{BER}=10^{-2}$ and $\mathrm{BER}=10^{-5}$, respectively. We normalize the power with MRC scheme when the spectral efficiency equals to 1. Each user is assumed to have the same desired time-average throughput $T_{1}^{\text {ave }}=\ldots=T_{K}^{\text {ave }}$. Define window size $=\left(T_{i}^{\max }-T_{i}^{\text {ave }}\right)=\left(T_{i}^{\text {ave }}-T_{i}^{\min }\right)=2 \mathrm{bit} / \mathrm{s}$, $\forall i$. Each user is assumed to have unit bandwidth. From the 


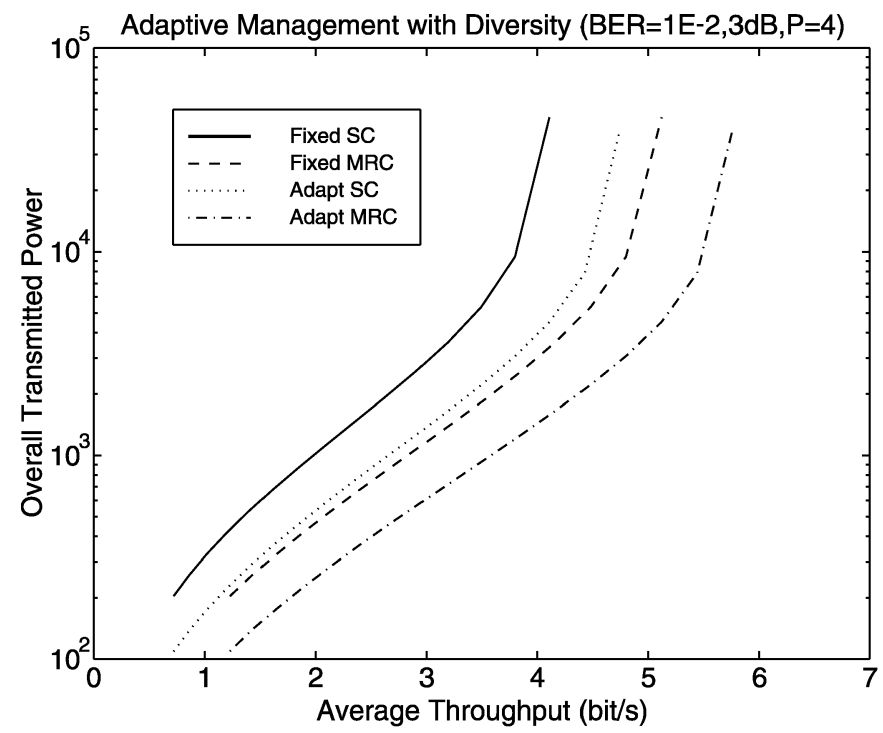

(a)

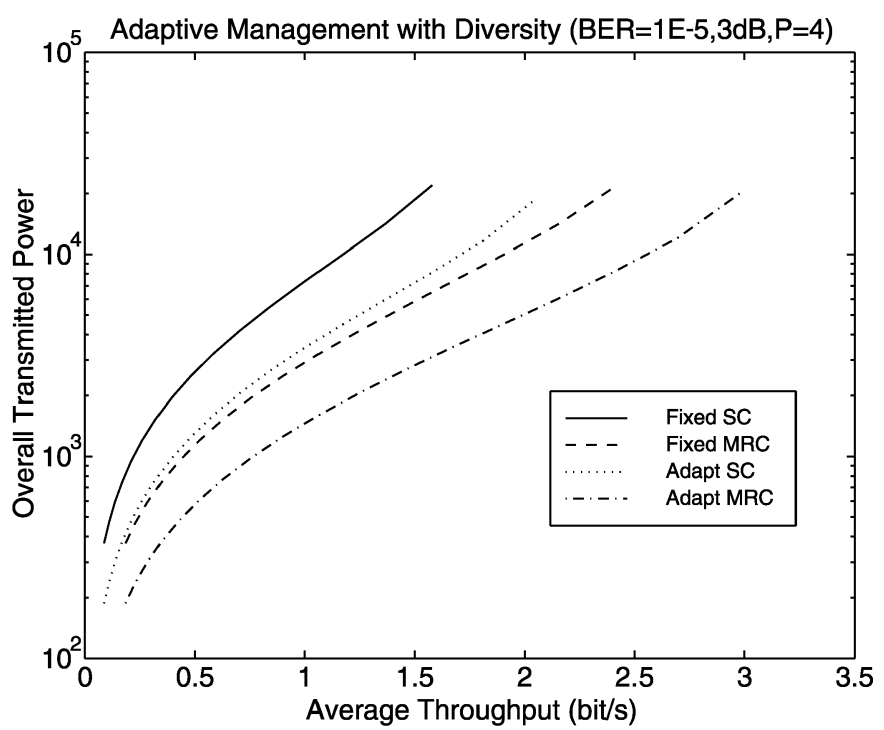

(b)

Fig. 6. MQAM performance with continuous throughput assumption. (a) BER $=10^{-2}$. (b) $\mathrm{BER}=10^{-5}$.

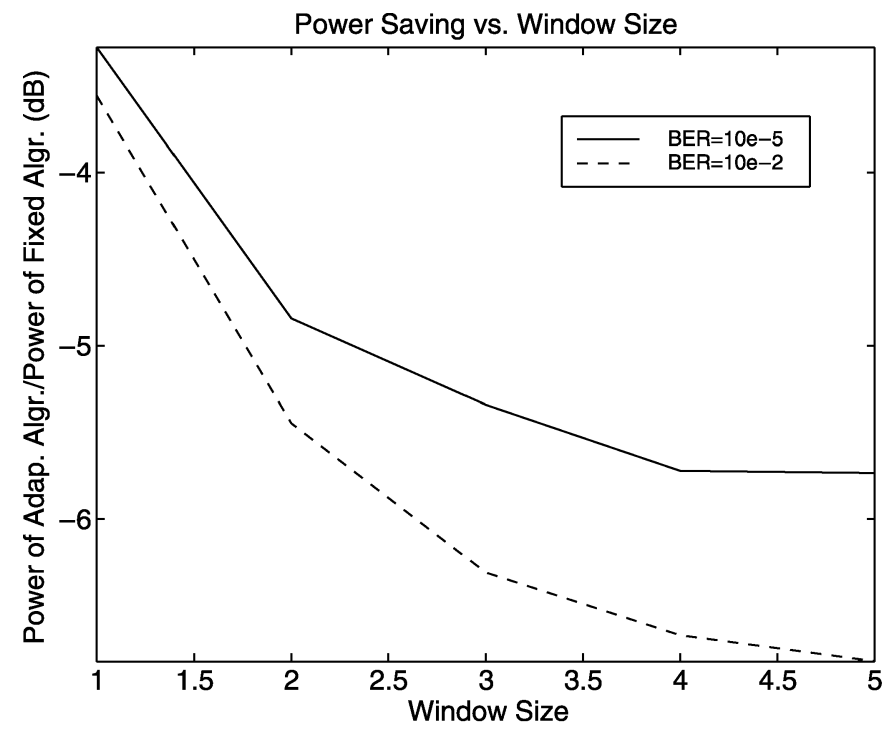

(a)

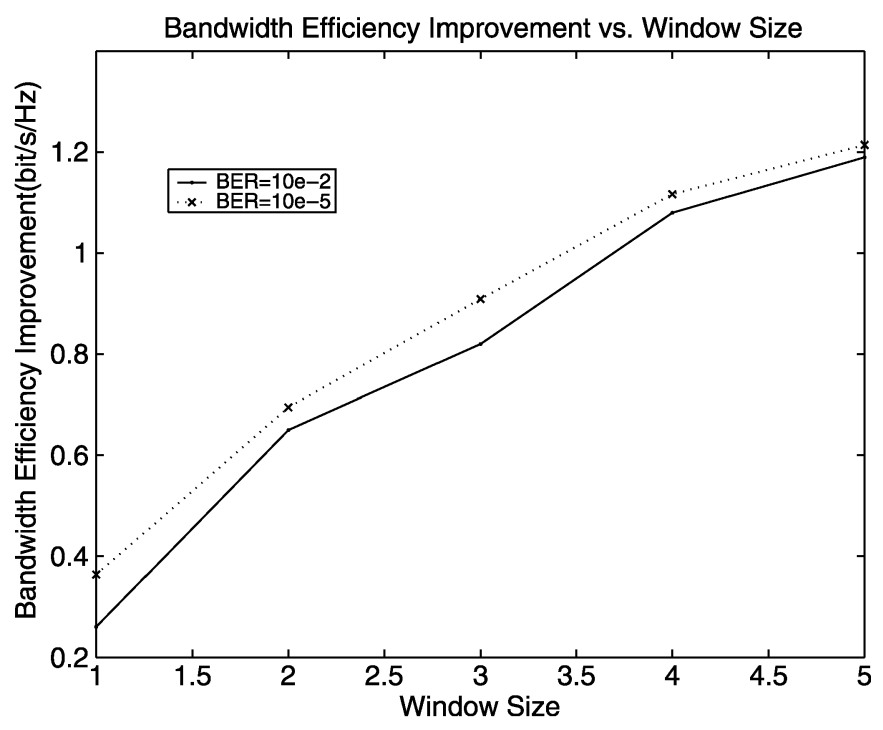

(b)

Fig. 7. Effects of window size: (a) power saving and (b) spectral efficiency gain.

simulation results, the projected gradient algorithm can find the optimal solution obtained by the full search algorithm. Because there is only one allocation scheme available when the average spectral efficiency is equal to one, all the algorithms perform the same. When the average spectral efficiency increases, the proposed algorithms greatly reduce the overall transmitted power and increase the maximum achievable throughput. The suboptimal fast search algorithm has the performance between those of the fixed scheme and optimal scheme. The results show that the proposed scheme can reduce the overall transmitted power by about $7 \mathrm{~dB}$, when the average spectral efficiency is larger than 2. The proposed scheme also increases the maximum spectral efficiency by about $1 \mathrm{bit} / \mathrm{s} / \mathrm{Hz}$. In the lower spectral efficiency range, the suboptimal fast search algorithm has almost the same performance as that of the optimal solution.
If the MRC diversity is employed, it reduces about 3 to 4 $\mathrm{dB}$ more transmitted powers than those of SC diversity. The SC diversity and proposed suboptimal algorithm have a lower complexity.

In order to further study the projected gradient method, the throughput is assumed to be continuous. In Fig. 6(a) and (b), the MQAM performances are compared with MRC and SC diversity at $\mathrm{BER}=10^{-2}$ and $\mathrm{BER}=10^{-5}$, respectively. The simulations are conducted from time 1 to 1000. From the results, it is shown that the adaptive algorithms can improve the average spectral efficiency by $0.9 \mathrm{bit} / \mathrm{s} / \mathrm{Hz}$ and decrease the overall transmitted power by $40 \%$ less than those of the fixed schemes. The MRC scheme again has better performance than SC scheme. The overall transmitted power of MRC is $40 \%$ less than that of SC. The maximum achievable spectral 
efficiency of MRC is about 0.7 to $0.9 \mathrm{bit} / \mathrm{s} / \mathrm{Hz}$ higher than that of SC. However, this improvement is decreasing as BER is getting smaller.

In Fig. 7(a) and (b), the average power saving and average spectral efficiency gain are shown as the functions of window size. The overall transmitted power can be reduced up to $7 \mathrm{~dB}$, and the spectral efficiency can be increased up to $1.2 \mathrm{bit} / \mathrm{s} / \mathrm{Hz}$. The power stops decreasing and spectral efficiency increasing speed is reduced, as the window size is growing. This is because of the time-average throughput constraint for each user. The user that gets better throughput at this time must pay back in the future. So, there is no need to have a very large window size. Only a limited number of modulation rates are necessary; consequently, the system complexity can be simple.

\section{CONCLUSION}

A joint power and throughput optimization framework is proposed to study the performance of adaptive resource allocation in wireless networks. The adaptive power minimization algorithms are constructed under the fairness constraint, by using adaptive modulation with antenna diversity to fully utilize the spectrum, to combat time-varying wireless channels and to reduce CCI. The proposed scheme can be interpreted as "water filling" each user's throughput in time domain and allocating the network throughput to different users each time. A joint power and throughput management system is built to adaptively allocate the resources. From the simulation results, the algorithms reduce the total transmitted power of mobile users by up to $7 \mathrm{~dB}$, which is critical in terms of battery life. The spectral efficiency is increased by up to $1.2 \mathrm{bit} / \mathrm{s} / \mathrm{Hz}$, which, in turn, increases the network performance.

\section{REFERENCES}

[1] S. Grandhi, R. Yates, and D. Goodman, "Resource allocation for cellular radio systems," IEEE Trans. Veh. Technol., vol. 46, pp. 581-588, Aug. 1997.

[2] R. Yates, "A framework for uplink power control in cellular radio systems," IEEE J. Select. Areas Commun., vol. 13, pp. 1341-1348, Sep. 1995.

[3] J. Zander and S. L. Kim, Radio Resource Management for Wireless Networks. Boston, MA: Artech, 2001.

[4] G. J. Foschini and Z. Miljanic, "A simple distributed autonomous power control algorithm and its convergence," IEEE Trans. Veh. Technol., vol. 42, pp. 641-646, Nov. 1993.

[5] F. Rashid-Farrokhi, L. Tassiulas, and K. J. R. Liu, "Joint optimal power control and beamforming in wireless network using antenna arrays," IEEE Trans. Commun., vol. 46, pp. 1313-1323, Oct. 1998.

[6] F. Rashid-Farrokhi, K. J. R. Liu, and L. Tassiulas, "Transmit beamforming and power control for cellular wireless systems," IEEE J. Select. Areas Commun., vol. 16, pp. 1437-1449, Oct. 1998.

[7] Y. C. Liang, F. P. S. Chin, and K. J. R. Liu, "Downlink beamforming for DS-CDMA mobile radio with multimedia services," IEEE Trans. Commun., vol. 49, pp. 1288-1298, July 2001.

[8] A. Yener, R. D. Yates, and S. Ulukus, "Interference management for cdma systems through power control, multiuser detection and beamforming," IEEE Trans. Commun., vol. 49, pp. 1227-1239, July 2001.

[9] W. T. Webb and R. Steele, "Variable rate QAM for mobile radio," IEEE Trans. Commun., vol. 43, pp. 2223-2230, July 1995.
[10] S. T. Chung and A. J. Goldsmith, "Degrees of freedom in adaptive modulation: A unified view," IEEE Trans. Commun., vol. 49, pp. 1561-1571, Sept. 2001.

[11] X. Tang, M. S. Alouini, and A. J. Goldsmith, "Effect of channel estimation error on M-QAM BER performance in Rayleigh fading," IEEE Trans. Commun., vol. 47, pp. 1856-1864, Dec. 1999.

[12] M. S. Alouini and A. J. Goldsmith, "A unified approach for calculating error rates of linearly-modulated signals over generalized fading channels," IEEE Trans. Commun., vol. 47, pp. 1324-1334, Sept. 1999.

[13] S. Falahati, A. Svensson, T. Ekman, and M. Sternad, "Adaptive modulation systems for predicted wireless channels," in Proc. 7th Wireless World Res. Forum Workshop, Eindhoven, Netherlands, Dec. 2002.

[14] _ _ "Effect of prediction errors on adaptive modulation systems for wireless channels," in Proc. Radiovetenskap och Kommunikation, Stockholm, Sweden, Jun. 2002, pp. 234-238.

[15] L. Hanzo, C. H. Wong, and M. S. Yee, Adaptive Wireless Transceivers: Turbo-coded Turbo-Equalized and Space-Time Coded TDMA, CDMA, and OFDM Systems. New York: Wiley, 2002.

[16] P. Ormeci, X. Liu, D. L. Goeckel, and R. D. Wesel, "Adaptive bit-interleaved coded modulation," IEEE Trans. Commun., vol. 49, pp. 1572-1581, Sept. 2001.

[17] K. J. Hole, H. Holm, and G. E. Oien, "Adaptive multidimensional coded modulation over flat fading channels," IEEE J. Select. Areas Commun., vol. 18, pp. 1153-1158, July 2000.

[18] D. Tse and S. Hanly, "Multi-access fading channels: Part I: Polymatroid structure, optimal resource allocation and throughput capacities," IEEE Trans. Inform. Theory, vol. 44, no. 7, pp. 2796-2815, Nov. 1998.

[19] T. M. Cover and J. A. Thomas, Elements of Information Theory. New York: Wiley, 1991.

[20] A. Ephremides and B. Hajek, "Information theory and communication networks: An unconsummated union," IEEE Trans. Inform. Theory, vol. 44, no. 6, pp. 2416-2434, 1998.

[21] Z. Han and K. J. R. Liu, "Joint adaptive power and modulation management in wireless networs with antenna diversity," in Proc. Sam2002, Rosslyn, VA, Aug. 2002.

[22] X. Qiu and K. Chawla, "On the performance of adaptive modulation in cellular systems," IEEE Trans. Commun., vol. 47, pp. 884-895, June 1999.

[23] K. K. Leung and L. C. Wang, "Controlling QoS by integrated power control and link adaptation in broadband wireless networks," Eur. Trans. Telecommun., no. 4, pp. 383-394, July 2000.

[24] J. S. Blogh and L. Hanzo, "Adaptive modulation and adaptive antenna array assisted network performance of multi-user detection aided UTRA-like FDD/CDMA systems," in Proc. VTC'2002, Vancouver, Canada, 2002, pp. 1806-1810.

[25] S. L. Kim, Z. Rosberg, and J. Zander, "Combined power control and transmission rate selection in cellular networks," in Proc. VTC, Amsterdam, The Netherlands, Fall 1999, pp. 1653-1657.

[26] Z. Han and K. J. R. Liu, "Adaptive SINR threshold allocation for joint power control and beamforming over wireless network," Proc. IEEE VTC, pp. 1548-1552, Fall 2001.

[27] _ - "Power minimization under constant throughput constraint in wireless networks with beamforming," in Proc. IEEE VTC, Vancouver, Canada, Sept. 2002.

[28] S. Kandukuri and S. Boyd, "Optimal power control in interference-limited fading wireless channels with outage-probability specifications," IEEE Trans. Wireless Commun., vol. 1, pp. 44-55, Jan. 2001.

[29] X. Liu, E. K. P. Chong, and N. B. Shroff, "Opportunistic transmission scheduling with resource-sharing constraints in wireless networks," IEEE Trans. Commun., vol. 48, pp. 1769-1773, Nov. 2000.

[30] C. U. Saraydar, N. B. Mandayam, and D. J. Goodman, "Efficient power control via pricing in wireless data networks," IEEE Trans. Commun., vol. 50, pp. 291-303, Feb. 2002.

[31] W. C. Jakes, Microwave Mobile Communications, 2nd ed. Piscataway, NJ: IEEE, 1994.

[32] M. Mesbahi, M. G. Safonov, and G. P. Papavassilopoulos, "Bilinearity and complementarity in robust control," in Advances in Linear Matrix Inequality Methods in Control. Philadelphia, PA: SIAM, 2000.

[33] P. Harley, "Short distance attenuation measurements at $900 \mathrm{MHz}$ and $1.8 \mathrm{GHz}$ using low antenna heights for microcells," IEEE Trans. Select. Areas Commun., vol. 37, pp. 220-222, Nov. 1988.

[34] G. Golub, Matrix Computations, 3rd ed. Baltimore, MD: Johns Hopkins Univ. Press, 1996. 


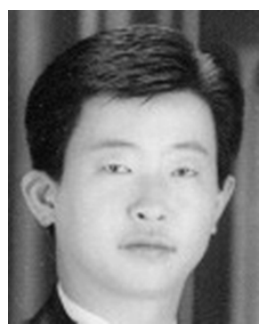

Zhu Han (S'01-M'04) received the B.S. degree in electronic engineering from Tsinghua University, in 1997, and the M.S. and Ph.D. degrees in electrical engineering from the University of Maryland, College Park, in 1997 and 2003, respectively.

From 1997 to 2000, he was a Graduate Research Assistant at the University of Maryland. From 2000 to 2002, he was an Engineer in the R\&D Group of ACTERNA, Maryland. He is currently a Research Associate at the University of Maryland. His research interests include wireless resource allocation and management, wireless communications and networking, game theory, and wireless multimedia.

Dr. Han is a member of the Technical Programming Committee for the IEEE International Conference on Communications of 2004 and 2005, the IEEE Vehicular Technology Conference, Spring 2004, the IEEE Consumer Communications and Networking Conference 2005, the IEEE Wireless Communications and Networking Conference 2005, and the IEEE Globe Communication Conference 2005, as well as Session Chair of the IEEE Wireless Communications and Networking Conference 2004.

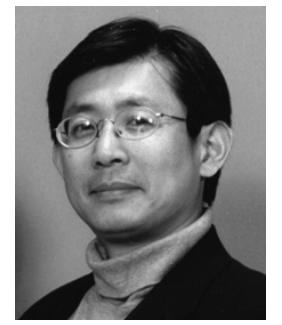

K. J. Ray Liu (S'87-M'90-SM'93-F'03) received the B.S. degree from the National Taiwan University and the Ph.D. degree from the University of California, Los Angeles, in 1983 and 1990, respectively, both in electrical engineering.

$\mathrm{He}$ is a Professor in the Electrical and Computer Engineering Department and Institute for Systems Research, University of Maryland, College Park. His research interests include signal processing algorithms and architectures; multimedia communications and signal processing; wireless communications and networking; information security; and bioinformatics, in which he has published over 300 refereed papers.

Dr. Liu is the recipient of numerous honors and awards including IEEE Signal Processing Society 2004 Distinguished Lecturer, the 1994 National Science Foundation Young Investigator Award, the IEEE Signal Processing Society's 1993 Senior Award (Best Paper Award), the IEEE 50th Vehicular Technology Conference Best Paper Award, Amsterdam, The Netherlands, 1999. He also received the George Corcoran Award in 1994 for outstanding contributions to electrical engineering education and the Outstanding Systems Engineering Faculty Award in 1996 in recognition of outstanding contributions in interdisciplinary research, both from the University of Maryland. He is the Editor-in-Chief of the IEEE Signal Processing Magazine and was the founding Editor-in-Chief of EURASIP Journal on Applied Signal Processing. He has served as an Associate Editor for the IEEE TRANSACTIONS ON Signal PROCEsSING, a Guest Editor of special issues on Multimedia Signal Processing in the PROCEEDINGS OF THE IEEE, a Guest Editor of a special issue on Signal Processing for Wireless Communications in the IEEE JOURNAL OF SELECTED AREAS IN COMMUNICATIONS, a Guest Editor of a special issue on Multimedia Communications over Networks in the IEEE Signal Processing Magazine, and a Guest Editor of a special issue on Multimedia over IP in the IEEE TRANSACTIONS ON Multimedia. He is a member of the Board of Governors of the IEEE Signal Processing Society. 